\title{
Gary/Ajar: la mort du double auteur
}

\section{Stéphane Maffli}

\section{(2) OpenEdition}

Journals

Édition électronique

URL : http://journals.openedition.org/edl/2406

DOI : 10.4000/edl.2406

ISSN : 2296-5084

\section{Éditeur}

Université de Lausanne

\section{Édition imprimée}

Date de publication : 15 mars 2020

Pagination : 151-154

ISBN : 978-2-940331-73-4

ISSN : 0014-2026

\section{Référence électronique}

Stéphane Maffli, «Gary/Ajar: la mort du double auteur », Études de lettres [En ligne], 312 | 2020, mis en ligne le 24 mars 2020, consulté le 17 septembre 2020. URL : http://journals.openedition.org/edl/2406 ; DOI : https://doi.org/10.4000/edl.2406

(c) Études de lettres 


\section{GARY/AJAR: LA MORT DU DOUBLE AUTEUR}

Le 2 décembre 1980, âgé de 66 ans, Romain Gary déjeune avec son éditeur Claude Gallimard, puis il rentre chez lui, rue du Bac à Paris, et se tire une balle dans la bouche. Il laisse une lettre qui se termine par ces mots: "Je me suis enfin exprimé entièrement.» ${ }^{1}$

Lauteur en question s'appelle d'abord Roman Kacew et naît en 1914. Dans La promesse de l'aube (1960), il raconte son enfance à Vilnius, son adolescence à Nice et son parcours d'aviateur au sein des Forces aériennes françaises libres. Avant la fin de la guerre, il publie sous le nom de Romain Gary son premier roman, Éducation européenne, sur la résistance dans les forêts polonaises, telle qu'il l'imagine. C'est avec Les racines du ciel (prix Goncourt 1956) qu'il devient un auteur de premier plan.

En 1973, un roman paraît sous le pseudonyme d'Émile Ajar. Le deuxième livre publié sous ce nom, La vie devant soi, reçoit le prix Goncourt en 1975. Or, un auteur ne pouvait recevoir ce prix qu'une seule fois. On ne soupçonne pas alors que Gary se cache derrière Ajar. Entre 1974 et 1980, l'écrivain publie sept romans, trois en tant que Romain Gary et quatre en tant qu'Émile Ajar.

L'affaire Ajar est complexe et profondément tragique. Le suicide de Gary, suivi de la révélation quelques mois plus tard qu'il est l'auteur des textes d'Émile Ajar et l'instigateur de ce qui est d'abord perçu comme une supercherie littéraire unique en son genre, me semblent être des événements assez importants pour contribuer à marquer, du point de vue de l'histoire littéraire, «l'an zéro du monde contemporain», car le contexte

I. La lettre est reproduite dans M. Decout, Album Romain Gary, p. 203. 
éditorial «Émile Ajar» fait partie de l'œuvre. Dans «Vie et mort d'Émile Ajar» (1981), bref texte posthume où il explique les raisons qui l'ont poussé à concevoir Ajar, Romain Gary indique qu'il n'y a que le poète écossais James Macpherson qui ait entrepris une imposture analogue, en imaginant la figure du barde Ossian dont il aurait traduit les textes du gaélique.

Reprenons. En 1973, Gary rédige Gros-Câlin, un roman sur la solitude et l'extrême timidité d'un narrateur qui a la particularité d'avoir un python pour animal de compagnie. La mue de son reptile le fascine. Au fil du livre, le python devient de plus en plus humain, et le narrateur s'identifie de plus en plus intensément à son serpent. Faire peau neuve au sens propre, changer d'identité, c'est un fantasme constant chez Gary, «le caméléon » 2 .

C'est après avoir terminé la rédaction de Gros-Câlin que Gary décide de le publier sous pseudonyme. Il sentait "qu'il y avait incompatibilité entre la notoriété, les poids et mesures selon lesquels on jugeait [son] œuvre, "la gueule qu'on [lui] avait faite" et la nature même du livre» ${ }^{3}$. Gary fait envoyer le manuscrit aux éditions Gallimard par un ami depuis le Brésil. L'auteur de Gros-Câlin aurait des problèmes avec la justice en France ce qui explique sa discrétion. «Émile Ajar» est donc à la base conçu comme le pseudonyme d'un homme au passé criminel et romanesque. C'est un jeu de poupées russes, analogue aux montages financiers avec plusieurs sociétés écran. Sauf qu'ici, ce n'est pas d'argent non déclaré qu'il s'agit, mais d'une œuvre non déclarée.

Quand le manuscrit arrive chez Gallimard, Raymond Queneau fait partie du comité de lecture ${ }^{4}$. On décline le manuscrit considéré comme un premier roman, mais on le recommande au Mercure de France, alors dirigé par Simone Gallimard.

À la sortie de Gros-Câlin, le succès commercial et critique est au rendez-vous, et Gary concrétise en quelque sorte ce que Roland Barthes avait préconisé pour la critique quelques années plus tôt dans «La mort de l'auteur ": L'écriture "est destruction de toute voix, de toute origine. L'écriture, c'est ce neutre, ce composite, cet oblique où fuit notre sujet, le noir-et-blanc où vient se perdre toute identité, à commencer par celle-là

2. Voir M. Anissimov, Romain Gary, le caméléon.

3. R. Gary, "Vie et mort d'Émile Ajar», in R. Gary, Romans et récits, t. II, p. 1432.

4. Voir la lettre reproduite dans M. Decout, Album Romain Gary, p. 155. 
même du corps qui écrit” ${ }^{5}$. Avec Gros-Câlin, Romain Gary avait libéré son œuvre de lui-même. La figure de l'auteur ne disparaît certes pas complètement, mais elle devient abstraite, inventée: elle change de nature. Il est certain que la critique parisienne aurait jugé le livre différemment si elle avait su quel auteur se cachait derrière.

Pour les trois romans suivants, Romain Gary demande à Paul Pavlowitch, né en 1942 et fils de sa cousine germaine, de «jouer» Émile Ajar. Il s'agit d'incarner l'auteur auprès de l'éditeur et de la presse pour le deuxième roman, La vie devant soi. Paul a "la tête de l'emploi: sauvage, atypique, baroudeur " ${ }^{6}$. Mais il prend des libertés par rapport à ce que Gary lui demande de faire. À la différence d'un personnage fictif, malléable à souhait jusqu'au moment de la publication du roman, le personnage réel à qui Gary demande d'incarner Ajar ne se trouve pas être le comédien docile dont il aurait besoin.

Le 30 septembre 1975, à Copenhague, Paul rencontre pour un entretien Yvonne Baby, la journaliste en charge de la culture au Monde, et prend des libertés par rapport aux indications du cousin de sa mère. Il glisse aussi à l'éditeur une photo de lui-même. Un journaliste du Point retrouve sa trace et «découvre le nom de celui qui, jusqu'alors, ne prêtait à Ajar que son visage " ${ }^{7}$. Le lien familial avec Romain Gary est établi et les soupçons sur la paternité de l'œuvre d'Ajar se font de plus en plus forts ${ }^{8}$. La relation entre l'auteur qui écrit et la personne qui l'incarne s'envenime; elle devient même l'objet du troisième roman signé Ajar, Pseudo, qui sort le 15 novembre 1976 toujours au Mercure de France. Une fois de plus, la frontière entre fiction et réalité est dissoute: se présentant comme «une autofiction de Paul Pavlowitch écrite sous le nom

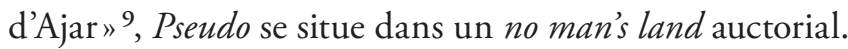

Le suicide de Romain Gary marque la fin de l'affaire Ajar. Le masque était-il devenu trop lourd à porter? Comme tout suicide, l'acte dans sa radicalité laisse perplexe. Gary s'était enfermé dans le mensonge; la situation de celui qui avait inventé l'auteur de ses romans était devenue intenable.

5. R. Barthes, «La mort de l'auteur», p. 61.

6. M. Decout, Album Romain Gary, p. 163.

7. Ibid., p. 170.

8. Pour le déroulement de toute l'affaire, on se référera à M. Decout (p. 154-206), à M. Anissimov (p. 411-659) et aux notices de Romans et récits, t. II.

9. M. Decout, Album Romain Gary, p. 176. 
L'affaire Ajar est en ceci fascinante qu'elle questionne le rapport entre le monde du texte et le monde réel. Romain Gary a su ouvrir une brèche entre la fictionnalité et la factualité comme personne ne l'avait fait avant lui. "Ajar» est bien plus qu'un pseudonyme ou un prête-nom: à défaut d'être une personne physique, "Ajar» est un personnage littéraire hors du commun, une ouvre artistique et littéraire totale qui va au-delà du texte.

\author{
Stéphane MAfFli
}

Section d'allemand, Faculté des lettres, Université de Lausanne

\title{
BIBLIOGRAPHIE
}

Anissimov, Myriam, Romain Gary, le caméléon, Paris, Denoël, 2006.

Barthes, Roland, "La mort de l'auteur" (1968), in Le Bruissement de la langue, Paris, Seuil, 1984, p. 61-67.

Decout, Maxime, Album Romain Gary, Paris, Gallimard, 2019 (Bibliothèque de la Pléiade).

Gary, Romain, Romans et récits, 2 t., Paris, Gallimard, 2019 (Bibliothèque de la Pléiade). 